\title{
Relation of the polymorphism of cyp51A sequence and the susceptibility of Aspergillus fumigatus isolates to triazoles determined by commercial gradient test (Etest) and by reference methods
}

\author{
Urszula Nawrot ${ }^{\boxplus}$, Beata Sulik-Tyszka2,3, Ewelina Kurzyk ${ }^{4}$, Martyna Mroczyńska4, \\ Katarzyna Włodarczyk ${ }^{1}$, Marta Wróblewska ${ }^{2,3}$, Grzegorz Władysław Basak ${ }^{5}$ and \\ Anna Brillowska-Dąbrowska ${ }^{4}$
}

'Department of Pharmaceutical Microbiology and Parasitology, Faculty of Pharmacy, Wrocław Medical University, Wrocław, Poland; ${ }^{2}$ Department of Microbiology, Central Clinical Hospital, Warsaw Medical University, Warsaw, Poland; ${ }^{3}$ Department of Dental Microbiology, Warsaw Medical University, Warsaw, Poland; ‘ Department of Molecular Biotechnology and Microbiology, Gdańsk University of Technology, Gdańsk, Poland; 5 Department of Haematology, Oncology and Internal Medicine, Medical University of Warsaw, Warsaw, Poland

The aim of this study was to evaluate the accuracy of commercial gradient test (Etest) in the detection of triazole resistant Aspergillus fumigatus isolates using reference microdilution methods and the analysis of sequences of the cyp 51A gene. The study was performed on twenty clinical isolates which were identified as Aspergillus fumigatus based on the DNA sequences of the ITS1-2 fragment of ribosomal DNA and the $\beta$-tubulin gene, out of them seventeen isolates showed wild-type cyp51A sequence and three were positive for the mutation TR34/L98H. All isolates were tested for the susceptibility to itraconazole (ITZ), voriconazole (VOR) and posaconasole (POS) using microdilution methods, according to EUCAST and CLSI protocols, as well as using Etest. The results of microdilution and Etests were analysed separately according to clinical breakpoints (CBP) defined by EUCAST version 7.0 and epidemiological cut off values (ECV). Etest as well as reference methods excellently recognised the WT isolates, which were susceptible to all tested triazoles, regardless of the method and CBP or ECV criteria used. The Etest recognized three non-WT isolates as resistant or intermediately sensitive to ITZ and POS and one as resistant to VOR. The categorical concordance between Etests and EUCAST and Etests and the CLSI method ranged from 90 to $100 \%$. The interpretation of the results obtained from routine $A$. fumigatus Etests requires great caution. The use of the confirmative examinations with reference AST methods as well as with molecular tests is recommended.

Key words: Aspergillus fumigatus, triazole resistance, susceptibility testing, Etest, cyp51A sequence

Received: 23 March, 2017; revised: 02 June, 2017; accepted: 23 August, 2017; available on-line: 30 November, 2017

e-mail: e-mail: urszula.nawrot@umed.wroc.pl

Abbreviations: ITZ, itraconazole; VOR, voriconazole; POS, posaconazole; CBP, clinical breakpoints; ECV, epidemiological cut off valᄀue; MIC, minimal inhibitory concentration; AST, antifungal susceptibility testing: CLSI, Clinical and Laboratory Standards Institute ICU, intensive care unit; EUCAST, The European Committee on Antimicrobial Susceptibility Testing; CFU, colony forming unit; S, susceptible; R, resistant; I, intermediately susceptible; WT, wild-type ATCC, American Type Culture Collection; ITS, internal transcribed spacer; TR, tandem repeats; EORTC, European Organization for Research and Treatment of Cancer.

\section{INTRODUCTION}

Among fungi, Aspergillus plays an increasingly important role as a human and animal pathogen responsible for infections as well as allergies. Due to the ubiquitous character of fungal spores and its ability to easily disperse in the air, the respiratory tract is the most frequent port of entry and subsequently place of the disease development. The spectrum of antifungals suitable for treatment of aspergillosis includes triazole derivatives voriconazole (VOR), itraconazole (ITZ) and posaconazole (POS) as well as amphotericine $\mathrm{B}$, representing polyenes. Aspergillus fumigatus, which currently is the most frequent filamentous fungus isolated from infection worldwide, shows an innate susceptibility to both triazoles and amphotericine B (Drgona et al., 2014). Taking into account pharmacokinetic properties and serious adverse effects connected with the implementation of amphotericine B, triazoles are recommended as a first line therapy for aspergillosis (Drgona et al., 2014; Groll et al., 2014; Maertens et al., 2011). An alarming phenomenon of acquired resistance of Aspergillus fumigatus to triazoles has recently been reported worldwide (Arendrup, 2014). Main mechanism of triazole resistance relies on mutations in target cyp $51 \mathrm{~A}$ gene and/or in its promoter region and subsequently on substitution of amino acids in lanosterol 14 alpha-demethylase (protein CYP 51A). In Europe, 3.2\% of clinical Aspergillus fumigatus isolates are resistant to triazoles and approximately 50\% of them display mechanisms TR34/L98H (34bp tandem repeats incorporated in the promotor region and single point mutation of cyp $51 \mathrm{~A}$ gene resulting in the substitution of leucine for histidine) (van der Linden et al., 2015). This mechanism is also frequent among environmental isolates (0-26\%), which can be a source of infection in humans (Rodriguez-Tudela et al. 2008). This situation stresses the necessity to perform routine susceptibility tests. At present, two reference microdilution methods of susceptibility testing for filamentous fungi are available (Clinical and Laboratory Standards Institute, 2008; EUCAST DEFINITIVE DOCUMENT E Def 9.2 2014). Nevertheless, due to the complicated procedures, they are difficult to perform in clinical laboratories. Instead, the suitability 
of Etests in routine diagnostics has been indicated, but its applicability to moulds is still under discussion (Espinel-Ingroff \& Rezusta, 2002; Colosi et al., 2012).

The aim of this study was to evaluate the accuracy of Etests used in routine diagnostics to examine the susceptibility of clinical isolates of Aspergillus fumigatus to triazoles (VOR, ITZ and POS). As a comparison, the reference microdilution methods according to the European Committee for Antimicrobial Susceptibility Testing (EUCAST) (Arendrup et al., 2014) and Clinical and Laboratory Standards Institute (CLSI) (2008) were used. The susceptibility results were combined with the previously described sequences of the cyp $51 \mathrm{~A}$ gene encoding lanosterol 14 alphademethylase - the target for triazoles (Nawrot et al., 2017).

\section{MATERIAL AND METHODS}

Strains and study design. Twenty clinical isolates of Aspergillus fumigatus from the laboratory collection of the Department of Microbiology, Medical University of Warsaw (Poland) were selected for the study. The isolates were obtained in years 2007-2015 from patients with proven or probable aspergillosis according to EOTRC/ MSG criteria (each strain from another individual) (De Pauw et al., 2008). The patients were hospitalised in different departments including haematology -6 patients, pulmonology -6 , ICU -4 , nephrology -1 , cardiosurgery 1 , endocrinology -1 , and internal medicine -1 . Most isolates originated from respiratory tract samples (BAL -8 , sputum -5 , broncho-aspirate -3 ) and the rest were from the pericardial fluid -1 isolate, bone tissue -1 , blood -1 , and wound -1 . The strains were preserved by being frozen at $-86^{\circ} \mathrm{C}$ using the Microbank ${ }^{\mathrm{TM}}$ Preservation System (Pro-Lab Diagnostics ${ }^{\text {TM}}$ ) and before testing were revived by subculture on Sabouraud dextrose agar. The isolates were identified as Aspergillus fumigatus based on the morphology and sequencing of rDNA and beta-tubuline genes. Seventeen isolates represented wild -type cyp51A sequence and three were positive for the mutation TR34/ L98H (Table 1) (Nawrot et al., 2017).

Each strain was tested for the susceptibility to itraconazole (ITZ), voriconazole (VOR), and posaconazole (POS) by microdilution methods according to EUCAST (Arendrup et al., 2014) and CLSI (2008) and the obtained results were compared with Etest. In cases where significant discordance was observed between the applied methods, the Etests were performed again.

Susceptibility testing. Minimal Inhibitory Concentration (MIC) was determined with broth microdilution methods, according to EUCAST E.DEF 9.2 (Arendrup et al., 2014) and according to the M38-A2 Document of the Clinical and Laboratory Standards Institute (2008). The RPMI1640 medium, buffered with MOPS and supplemented with glucose $(2 \%$ in EUCAST and $0.2 \%$ in CLSI), was used to prepare inoculums of final density ranging $1-2.5 \times 10^{5} \mathrm{CFU} / \mathrm{mL}$ (EUCAST) or $0.4-5 \times 10^{4}$ $\mathrm{CFU} / \mathrm{mL}$ (CLSI). The $100 \mu \mathrm{l}$ aliquots of prepared cultures were inoculated to the flat-bottom wells of 96-wells microtiter plates containing $100 \mu \mathrm{l}$ of tested drug solutions (two-fold serial drug dilutions ranging $0.0156-8 \mathrm{mg} / \mathrm{L}$ ). The MIC was visually determined after $48 \mathrm{~h}$ of incubation at $35^{\circ} \mathrm{C}$ as the lowest drug concentration resulting in complete inhibition of fungal growth. All antifungals as well as main reagents (DMSO, RPMI 1640 medium, MOPS buffer) were obtained from Sigma-Aldrich.

The gradient susceptibility tests were performed using Etest-strips (BioMerieux, France) and RPMI 1640-2\% glucose agar plates (Biomed, Poland) according to the manufacturer's instructions. Tests were incubated at $35^{\circ} \mathrm{C}$ for up to $48 \mathrm{~h}$. The MIC values were indicated at the intersection of the inhibition zone with the Etest strips.

For the quality control of susceptibility tests, Candida krusei ATCC 6258 and C. parapsilosis ATCC 22019 were used.

The results of microdilution and Etests were analysed according to clinical breakpoints (CBP) defined by EUCAST version 7.0 (http://www.eucast.org/fileadmin/ src/media/PDFs/EUCAST_files/AFST/Antifungal_ breakpoints_v_7.0.pdf ) and epidemiological cut off values (ECV) served as breakpoints before ratified clinical breakpoints by CLSI (Espinel-Ingroff et al., 2010). According to EUCAST the isolates of Aspergillus fumigatus were reported as resistant when the MIC value was higher than $2 \mathrm{mg} / \mathrm{L}$ for ITZ and VOR and $0.25 \mathrm{mg} / \mathrm{L}$ for POS, and as susceptible when the MIC value was equal or lower than $1 \mathrm{mg} / \mathrm{L}, 1 \mathrm{mg} / \mathrm{L}$ and $0.12 \mathrm{mg} / \mathrm{L}$, respectively. Based on ECVs, the results of MIC classified the strains as resistant (non-WT) to ITZ, VOR, and POS when they exceeded $1 \mathrm{mg} / \mathrm{L}, 1 \mathrm{mg} / \mathrm{L}$ and $0.25 \mathrm{mg} / \mathrm{L}$, respectively. The strains with $\mathrm{MIC}$ lower than or equal to $1 \mathrm{mg} / \mathrm{L}, 1 \mathrm{mg} / \mathrm{L}$ and $0.25 \mathrm{mg} / \mathrm{L}$, respectively, were regarded as susceptible (W'T).

\section{RESULTS}

The results of susceptibility testing obtained by CLSI, EUCAST and Etest methods, as well as interpretation of susceptibility data and comparison of the methods are shown in Tables $1-3$. The MIC values determined by CLSI and EUCAST methods did not differ in more than one dilution for VOR and POS and two dilutions for ITZ (Table 3). For most of the tested isolates, differences be-

Table 1. Results of susceptibility tests for Aspergillus fumigatus isolates with wild type and the mutation TR34/L98H in the cyp51A sequence

\begin{tabular}{|c|c|c|c|c|c|c|c|c|c|}
\hline \multirow{3}{*}{$\begin{array}{l}\text { cyp51A sequence (num- } \\
\text { ber of strains) }\end{array}$} & \multicolumn{9}{|c|}{ Range of MIC mg/L (mean) } \\
\hline & \multicolumn{3}{|c|}{ Itraconazole } & \multicolumn{3}{|c|}{ Voriconazole } & \multicolumn{3}{|c|}{ Posaconazole } \\
\hline & CLSI & EUCAST & Etest & CLSI & EUCAST & Etest & CLSI & EUCAST & Etest \\
\hline WT $(n=17)$ & $\begin{array}{c}0.06-0.5 \\
(0.26)\end{array}$ & $\begin{array}{c}0.061-1 \\
(0.48)\end{array}$ & $\begin{array}{c}0.006-1 \\
(0.28)\end{array}$ & $\begin{array}{l}0.125-0.5 \\
(0.33)\end{array}$ & $\begin{array}{l}0.125-0.5 \\
(0.28)\end{array}$ & $\begin{array}{l}0.016-0.38 \\
(0.113)\end{array}$ & $\begin{array}{l}0.015-0.06 \\
(0.058)\end{array}$ & $\begin{array}{l}0.015-0.06 \\
(0.036)\end{array}$ & $\begin{array}{c}0.008-0.125 \\
(0.04)\end{array}$ \\
\hline \multicolumn{10}{|l|}{$\begin{array}{l}\text { TR34/L98H positive } \\
(n=3):\end{array}$} \\
\hline 'No 5287 & $>8$ & $>8$ & 1.5 & 2.0 & 1.0 & 1 & 0.5 & 0.5 & 0.38 \\
\hline No 2708 & $>8$ & $>8$ & 16 & 4.0 & 2.0 & 2.0 & 0.5 & 0.5 & 0.5 \\
\hline 'No 1473 & $>8$ & $>8$ & 3 & 1.0 & 2.0 & 0.75 & 0.25 & 0.5 & 0.38 \\
\hline
\end{tabular}

${ }^{1}$ results of Etests after $48 \mathrm{~h}$ incubation are presented; MICs obtained after $24 \mathrm{~h}$ incubation for ITR, VOR, POS were $0.5,0.125,0.125$ mg/L, respectively 
Table 2. Categorisation of susceptibility results based on epidemiological cut-off values (ECV) and clinical breakpoints (CBP) of EUCAST v7

\begin{tabular}{|c|c|c|c|c|c|c|c|c|c|}
\hline \multirow{3}{*}{$\begin{array}{l}\text { cyp51A sequence (number of } \\
\text { strains) }\end{array}$} & \multicolumn{9}{|c|}{ Categorisation according to ECV/CBP } \\
\hline & \multicolumn{3}{|c|}{ Itraconazole } & \multicolumn{3}{|c|}{ Voriconazole } & \multicolumn{3}{|c|}{ Posaconazole } \\
\hline & CLSI & EUCAST & Etest & CLSI & EUCAST & Etest & CLSI & EUCAST & Etest \\
\hline WT $(n=17)$ & $\mathrm{S} / \mathrm{S}$ & $\mathrm{S} / \mathrm{S}$ & $\mathrm{S} / \mathrm{S}$ & $\mathrm{S} / \mathrm{S}$ & $\mathrm{S} / \mathrm{S}$ & $\mathrm{S} / \mathrm{S}$ & $\mathrm{S} / \mathrm{S}$ & $\mathrm{S} / \mathrm{S}$ & $\mathrm{S} / \mathrm{S}$ \\
\hline \multicolumn{10}{|l|}{ TR34/L98H positive $(n=3)$ : } \\
\hline 'No 5287 & $R / R$ & $R / R$ & $\mathrm{R} / \mathrm{l}$ & $\mathrm{R} / \mathrm{l}$ & $S / S$ & $S / S$ & $R / R$ & $R / R$ & $R / R$ \\
\hline No 2708 & $R / R$ & $R / R$ & $R / R$ & $R / R$ & $\mathrm{R} / \mathrm{l}$ & $\mathrm{R} / \mathrm{l}$ & $R / R$ & $R / R$ & $R / R$ \\
\hline${ }^{1}$ No 1473 & $R / R$ & $\mathrm{R} / \mathrm{R}$ & $\mathrm{R} / \mathrm{R}$ & $S / S$ & $\mathrm{R} / \mathrm{l}$ & $S / S$ & $\mathrm{~S} / \mathrm{I}$ & $\mathrm{R} / \mathrm{R}$ & $R / R$ \\
\hline
\end{tabular}

1interpretation of Etests according to results obtained after $48 \mathrm{~h}$; categorisation according to MICs obtained after $24 \mathrm{~h}$ was: S/S for ITZ, VOR, as well as POS

tween MICs obtained with Etests and CLSI or EUCAST were not higher than two dilutions (except of five, six and one isolate, which showed a difference of $>2$ dilutions for ITZ, VOR, or POS, respectively). Most isolates $(17 / 20)$ showed wild-type cyp51A sequence and displayed susceptibility to all tested triazoles, regardless of the method used and of CLSI or EUCAST interpretative criteria. Some discrepancies in susceptibility results were found for three isolates, which were found to be positive for the mutation TR34/L98H. The MIC values obtained in reference and gradient tests for ITZ and POS against non-WT isolates were more similar than those obtained for VOR. Namely, the MIC of ITZ measured with CLSI and EUCAST was $>8 \mathrm{mg} / \mathrm{L}$ for isolates No 5287, 1473 and 2708, whereas the results of the Etest were $1.5,3$, and $16 \mathrm{mg} / \mathrm{L}$, respectively. Applying the CBP (EUCAST) allowed identification of one small categorical error (I instead of $\mathrm{R}$ ) between results of the Etest and both reference methods. The MIC of POS for the isolate No1473 obtained with the CLSI method $(0.25 \mathrm{mg} / \mathrm{L})$ was lower than with EUCAST and the E-test $(0.5 \mathrm{mg} / \mathrm{L})$, and as a consequence the categorical disagreement of the major ( $\mathrm{S} v s \mathrm{R}$, according to ECV) or minor ( $\mathrm{S}$ vs $\mathrm{I}$, according to $\mathrm{CBP}$ ) level was found. In the case of VOR, the concordant result of CLSI and EUCAST was found for one non-WT isolate (No 2708), whereas for isolates No 1473 and No 5287, the MIC value was $1.0 \mathrm{mg} / \mathrm{L}$ in the CLSI and EUCAST method, respectively (Table 1). In contrast, the Etest detected resistance of one strain (No 2708). However, when compared to results obtained by CLSI or EUCAST, the Etests were in agreement with results obtained for $2 / 3$ isolates. The overall categorical agreement between CLSI and EUCAST was $100 \%$ and $95 \%$ for ITZ and POS and $85-90 \%$ for VOR (depending on ECV or CBPs criterion). The concordance between Etests and EUCAST and Etests and the CLSI method ranged from 90 to 100\%. (Table 3).

\section{DISCUSSION}

Due to the complex morphological structure, moulds represent a difficult subject for laboratory examination. However, the development of the reference methods of susceptibility testing for filamentous fungi by CLSI and EUCAST represents major progress in mycological diagnostics. Despite the methods appearing to be similar (they varied only in the glucose concentration in the RPMI1640 medium and density of fungal inoculum), obtained results are sometimes inconsistent and the interpretative criteria must be developed individually for each method. The present study showed that although the correlation between the CLSI and EUCAST methods was very good (difference in $\leq 2$ dilutions for $100 \%$ isolates), the interpretation according to the CBPs published by EUCAST may differ substantially from those based on epidemiological cut-off values used by CLSI. The extensive comparison of the EUCAST and CLSI methods for testing activity of VOR, POS, and ITZ against Aspergillus spp was described by Pfaller and coworkers (2011). Similarly to our study, the authors found excellent agreement between the methods and obtained MIC results did not differ in more than 2 dilutions for 100-97.5\% of Aspergillus fumigatus isolates. Pfaller and coworkers (2011) categorised isolates as WT and non-WT, and found the highest number of discrepant results for POS. In the present study, applying the above mentioned criterion allowed us to find two major discrepancies ( $\mathrm{S} v s . \mathrm{R})$ for VOR and one for POS.

Currently, a very important problem for routine diagnostics is the reliability and categorisation of the results obtained by commercial tests, especially the gradient diffusion Etests. Because the Etest does not require advanced equipment and is easy to perform it represents very attractive alternative for the microdilution method in many diagnostic laboratories. In this study, we evaluated the results of the

\section{Table 3. Concordance in susceptibility results obtained with Etest and microdilution methods}

\begin{tabular}{|c|c|c|c|c|c|c|c|c|c|}
\hline & \multicolumn{3}{|c|}{$\begin{array}{l}\text { Number of strains with MIC differing in } \\
\pm 0 / 1 / 2 />2 \text { dilution* }\end{array}$} & \multicolumn{3}{|c|}{$\begin{array}{l}\text { Categorical agreement according to } \\
\text { ECVs }\end{array}$} & \multicolumn{3}{|c|}{$\begin{array}{l}\text { Categorical agreement according to } \\
\text { CBP }\end{array}$} \\
\hline & $\begin{array}{l}\text { CLSI vs } \\
\text { EUCAST }\end{array}$ & $\begin{array}{l}\text { Etest vs } \\
\text { CLSI }\end{array}$ & $\begin{array}{l}\text { Etest vs } \\
\text { EUCAST }\end{array}$ & $\begin{array}{l}\text { CLSI vs } \\
\text { EUCAST }\end{array}$ & $\begin{array}{l}\text { Etest vs } \\
\text { CLSI }\end{array}$ & $\begin{array}{l}\text { Etest vs } \\
\text { EUCAST }\end{array}$ & $\begin{array}{l}\text { CLSI vs } \\
\text { EUCAST }\end{array}$ & $\begin{array}{l}\text { Etest vs } \\
\text { CLSI }\end{array}$ & $\begin{array}{l}\text { Etest vs } \\
\text { EUCAST }\end{array}$ \\
\hline ITZ & $7 / 10 / 3 / 0$ & $9 / 4 / 2 / 5$ & $4 / 6 / 5 / 5$ & $100 \%$ & $100 \%$ & $100 \%$ & $100 \%$ & $95 \%$ & $95 \%$ \\
\hline VOR & $12 / 8 / 0 / 0$ & $3 / 9 / 2 / 6$ & $4 / 7 / 3 / 6$ & $18 / 20(90 \%)$ & $\begin{array}{l}18 / 20 \\
(90 \%)\end{array}$ & $\begin{array}{l}19 / 20 \\
(95 \%)\end{array}$ & $\begin{array}{l}17 / 20 \\
(85 \%)\end{array}$ & $\begin{array}{l}18 / 20 \\
(90 \%)\end{array}$ & $\begin{array}{l}18 / 20 \\
(90 \%)\end{array}$ \\
\hline POS & $7 / 13 / 0 / 0$ & $9 / 5 / 5 / 1$ & $9 / 8 / 2 / 1$ & $19 / 20$ (95\%) & $\begin{array}{l}19 / 20 \\
(95 \%)\end{array}$ & $100 \%$ & $\begin{array}{l}19 / 20 \\
(95 \%)\end{array}$ & $\begin{array}{l}19 / 20 \\
(95 \%)\end{array}$ & $100 \%$ \\
\hline
\end{tabular}

*the values of MIC by Etests were adjusted to two-fold dilutions used in the reference methods 
phenotypical antifungal susceptibility tests on a population of isolates with the defined sequence of the cyp51A gene. The isolates with the wild-type $y p 51 \mathrm{~A}$ sequence are likely to be susceptible to triazoles. The mutation TR34/L98H, which was detected in three isolates, represents the most frequent mechanism of azole-resistance in $A$. fumigatus and it is thought to be connected with cross-resistance to the Apergillus- active triazoles (Arendrup, 2014; Rodriguez-Tudela et al., 2008; van der Linden et al., 2015).

We found that ITZ and POS better indicate the TR34/ $\mathrm{L} 98 \mathrm{H}$ - positive isolates than VOR. Some isolates harbouring this mutation retain "in vitro" susceptibility to VOR, which in clinical practices may lead to overlooking the resistance. These data support the general rule described by Rodriguez-Tudela et al. (2008), that currently known $A$. fumigatus susceptible to ITR do not show resistance to other triazoles, and that routine antifungal susceptibility tests should always include ITZ. On the other hand, the resistance to ITZ could be a result of other mechanisms, e.g., upregulation of efflux pumps and does not necessarily indicate cross-resistance (Rodriguez-Tudela et al., 2008; Shapiro et al., 2011; Brillowska-Dabrowska et al., 2015). Another important observation described in the presented study is that the MIC values obtained in the Etest were often lower than in the reference methods and that an incubation time of $48 \mathrm{hrs}$ for the Etest improved detection of resistance. An impact of incubation time on the tests results was also discussed by other authors. According to Espinel-Ingroff \& Rezusta (2002) the $48 \mathrm{~h}$ incubation time of posaconazole Etests resulted in a better concordance with the reference method $(95 \%)$, while a better agreement for itraconazole MICs was after $24 \mathrm{~h}(90.3 \%)$. Due to a low number of isolates included in this study we were not able to reliably validate compared methods, however, observed disagreements indicate the necessity of farther studies in this field. The general agreement between Etests and the reference method is high. Nevertheless, taking into account some inconsistent results obtained for resistant isolates, the confirmative examinations with reference AST methods as well as with molecular tests are recommended.

\section{Conflict of interest}

U. Nawrot: received lecture honoraria and financial support for the participation in the congresses from Pfizer Polska Sp. z o.o.

All other authors declare that they have no conflict of interest.

\section{Acknowledgement}

This work was funded by the National Science Centre grant no. 2013/11/B/NZ7/04935.

\section{REFERENCES}

Arendrup MC (2014) Update on antifungal resistance in Aspergillus and Candida. Clin Microbiol Infect 20 Suppl 6: 42-48. doi: 10.1111/14690691.12513

Arendrup MC, Cuenca-Estrella M, Lass-Flörl C, Hope W, Howard S J and the Subcommittee on Antifungal Susceptibility Testing (AFST) of the ESCMID European Committee for Antimicrobial Susceptibility Testing (EUCAST). EUCAST DEFINITIVE DOCUMENT EDef 9.2 Method for the determination of broth dilution minimum inhibitory concentrations of antifungal agents for conidia forming moulds. July 2014. http://www.eucast.org/fileadmin/src/media/ PDFs/EUCAST_files/AFST/Files/EUCAST-AFST_EDEF_9_2_ Mould_testing_20140815.pdf

Brillowska-Dabrowska A, Mroczyńska M, Nawrot U, Włodarczyk K, Kurzyk E (2015) Examination of cyp51A and cyp51B expression level of the first Polish azole resistant clinical Aspergillus fumigatus isolate. Acta Biochim Pol 62: 837-839. doi: 10.18388/abp.2015_1143
Clinical and Laboratory Standards Institute (2008) Reference method for broth dilution antifungal susceptibility testing of filamentous fungi; approved standard, 2nd ed. CLSI document M38-A2. Clinical and Laboratory Standards Institute, Wayne, PA

Colosi IA, Faure O, Dessaigne B, Bourdon C, Lebeau B, Colosi HA, Pelloux H (2012) Susceptibility of 100 filamentous fungi: comparison of two diffusion methods, Neo-Sensitabs and E-test, for amphotericin B, caspofungin, itraconazole, voriconazole and posaconazole. Med Mycol 50: 378-385. doi: 10.3109/13693786.2011.616543

De Pauw B, Walsh TJ, Donnelly JP, Stevens DA, Edwards JE, Calandra T, Pappas PG, Maertens J, Lortholary O, Kauffman CA, Denning DW, Patterson TF, Maschmeyer G, Bille J, Dismukes WE, Herbrecht R, Hope WW, Kibbler CC, Kullberg BJ, Marr KA, Muñoz P, Odds FC, Perfect JR, Restrepo A, Ruhnke M, Segal BH, Sobel JD, Sorrell TC, Viscoli C, Wingard JR, Zaoutis T, Bennett JE; European Organization for Research and Treatment of Cancer/ Invasive Fungal Infections Cooperative Group; National Institute of Allergy and Infectious Diseases Mycoses Study Group (EORTC/MSG) Consensus Group. (2008) Revised definitions of invasive fungal disease from the European Organization for Research and Treatment of Cancer/Invasive Fungal Infections Cooperative Group and the National Institute of Allergy and Infectious Diseases Mycoses Study Group (EORTC/MSG) Consensus Group. Clin Infect Dis 46: 1813-1821. doi: 10.1086/588660

Drgona L, Khachatryan A, Stephens J, Charbonneau C, Kantecki M, Haider S, Barnes R. (2014) Clinical and economic burden of invasive fungal diseases in Europe: focus on pre-emptive and empirical treatment of Aspergillus and Candida species. Eur J Clin Microbiol Infect Dis 33: 7-21. doi: 10.1007/s10096-013-1944-3

Espinel-Ingroff A, Diekema DJ, Fothergill A, Johnson E, Pelaez T, Pfaller MA, Rinaldi MG, Canton E, Turnidge J (2010) Wild-type MIC distributions and epidemiological cutoff values for the triazoles and six Aspergillus spp. for the CLSI broth microdilution method (M38-A2 Document). J Clin Microbiol 48: 3251-3257. doi: 10.1128/ JCM.00536-10

Espinel-Ingroff A, Rezusta A (2002) E-test method for testing susceptibilities of Aspergillus spp. to the new triazoles voriconazole and posaconazole and to established antifungal agents: comparison with NCCLS broth microdilution method. I Clin Microbiol 40: 2101-2107

Groll AH, Castagnola E, Cesaro S, Dalle JH, Engelhard D, Hope W, Roilides E, Styczynski J, Warris A, Lehrnbecher T; Fourth European Conference on Infections in Leukaemia; Infectious Diseases Working Party of the European Group for Blood Marrow Transplantation (EBMT-IDWP); Infectious Diseases Group of the European Organisation for Research and Treatment of Cancer (EORTC-IDG); International Immunocompromised Host Society (ICHS); European Leukaemia Net (ELN). (2014) Fourth European Conference on Infections in Leukaemia (ECIL-4): guidelines for diagnosis, prevention, and treatment of invasive fungal diseases in paediatric patients with cancer or allogeneic haemopoietic stem-cell transplantation. Lancet $\mathrm{On}$ col 15: e327-340. doi: 10.1016/S1470-2045(14)70017-8

Maertens J, Marchetti O, Herbrecht R, Cornely OA, Flückiger U, Frêre P, Gachot B, Heinz WJ, Lass-Flörl C, Ribaud P, Thiebaut A, Cordonnier C; Third European Conference on Infections in Leukemia. (2011) European guidelines for antifungal management in leukemia and hematopoietic stem cell transplant recipients: summary of the ECIL 3- 2009 update. Bone Marrow Transplant 46: 709-718. doi: 10.1038/bmt.2010.175

Nawrot U, Kurzyk E, Arendrup MC,Mroczyńska M, Włodarczyk K, Sulik-Tyszka B, Wróblewska M, Ussowicz M, Zdziarski P, Niewińska K,Brillowska-Dąbrowska A (2017) Detection of Polish clinical Aspergillus fumigatus isolates resistant to triazoles. Med Mycol myx012. doi: $10.1093 / \mathrm{mmy} / \mathrm{myx} 012$

Pfaller M, Boyken L, Hollis R, Kroeger J, Messer S, Tendolkar S, Diekema D (2011) Comparison of the broth microdilution methods of the european committee on antimicrobial susceptibility testing and the clinical and laboratory standards institute for testing itraconazole, posaconazole, and voriconazole against Aspergillus isolates. J Clin Microbiol 49: 1110-1112. doi: 10.1128/JCM.02432-10

Rodriguez-Tudela JL, Alcazar-Fuoli L, Mellado E, Alastruey-Izquierdo A, Monzon A, Cuenca-Estrella M (2008) Epidemiological cutoffs and cross-resistance to azole drugs in Aspergillus fumigatus. Antimicrob Agents Chemother 52: 2468-2472. doi: 10.1128/AAC.00156-08

Shapiro RS, Robbins N, Cowen LE (2011) Regulatory circuitry governing fungal development, drug resistance, and disease. Microbiol Mol Biol Rev 75: 213-267. doi: 10.1128/MMBR.00045-10

van der Linden JW, Arendrup MC, Warris A, Lagrou K, Pelloux H, Hauser PM, Chryssanthou E, Mellado E, Kidd SE, Tortorano AM, Dannaoui E, Gaustad P, Baddley JW, Uekötter A, Lass-Flörl C, Klimko N, Moore CB, Denning DW, Pasqualotto AC, Kibbler C, Arikan-Akdagli S, Andes D, Meletiadis J, Naumiuk L, Nucci M, Melchers WJ, Verweij PE (2015) Prospective multicenter international surveillance of azole resistance in Aspergillus fumigatus. Emerg Infect Dis 21: 1041-1044. doi: 10.3201/eid2106.140717 